\title{
Malignant lymphoma of the uterine cervix presumptively diagnosed by Pap smear: A case report
}

\author{
TAKAHIRO KOYANAGI, HARUHIKO KONDO, ATSUSHI TOYAMA, MIHO ANDO, \\ SAKIKO IMAOKA, MASAYO INAMURA, HIROKO YAMAMOTO, SUMIE NAKAMURA, \\ YOKO TO, TATSUYA FUKAMI, MAKI GOTO, HIROSHI TSUJIOKA and FUYUKI EGUCHI \\ Department of Obstetrics and Gynecology, ASO lizuka Hospital, Iizuka, Fukuoka 820-8505, Japan
}

Received October 8, 2017; Accepted February 21, 2018

DOI: $10.3892 / \mathrm{ol} .2018 .8146$

\begin{abstract}
Malignant lymphoma of the uterine cervix is exceedingly rare and is difficult to diagnose by cervical cytology. The current study presents a case of malignant lymphoma of the uterine cervix that was presumptively diagnosed by cervical cancer screening in which the patient had no clinical symptoms. The anterior lip of the uterine cervix was occupied by a macroscopic hemorrhagic tumor. The obtained tumor cells exhibited typical cytological features of malignant lymphoma and were positive for CD20. The final diagnosis was diffuse large B cell lymphoma of the uterine cervix, stage IIEA (Ann Arbor classification). The patient received 6 courses of R-CHOP chemotherapy and achieved complete remission. Despite its rarity, the possibility of malignant lymphoma should be considered while screening for cervical cancers using Pap smears. The Pap test screening may be useful for the early diagnosis of malignant lymphoma of the uterine cervix in certain cases. By reaching a rapid and accurate diagnosis, immediate treatment may be initiated and surgery may be avoided.
\end{abstract}

\section{Introduction}

Malignant lymphoma of the uterine cervix and corpus is relatively rare and accounts for only $0.5 \%$ of extranodal lymphomas. The overall incidence of malignant lymphomas of the uterine cervix is less than $1 \%$ among all cervical malignancies (1), and only a few cases have been reported to date (2-4). It is difficult to diagnose malignant lymphomas of the uterine cervix by cervical cytology because lymphomas at this site are rare and can be misdiagnosed as other clinical conditions such as chronic cervicitis and epithelial malignancies (e.g., small cell carcinoma and poorly differentiated adenocarcinoma) (1).

Correspondence to: $\operatorname{Dr}$ Takahiro Koyanagi, Department of Obstetrics and Gynecology, ASO Iizuka Hospital, 3-83 Yoshio-machi, Iizuka, Fukuoka 820-8505, Japan

E-mail:koyataka@snow.plala.or.jp

Key words: B-cell lymphoma, cancer screening, chemotherapy, Papanicolaou test, uterine cervix
As a result, there are many cases where malignant lymphoma was diagnosed after invasive surgery.

Here, we present a case of malignant lymphoma of the uterine cervix presumptively diagnosed by the Papanicolaou (Pap) smear. We also discuss the clinicopathological features and therapeutic management of this rare tumor.

\section{Case report}

A 74-year-old woman (gravida 6, para 3) with no clinical symptoms had a medical checkup. Her past medical history was not significant. The result from the uterine cancer test was 'possible non-epithelial malignant tumor, including malignant lymphoma'. A detailed examination was conducted at our hospital.

Vaginal examination revealed a macroscopic, whitish hemorrhagic tumor (Fig. 1) occupying the anterior lip of the uterine cervix (Fig. 1A). Pelvic ultrasound showed an irregular round hypoechoic mass approximately $4 \mathrm{~cm}$ in diameter. Positron emission tomography (PET) scan demonstrated a uterine cervical mass with high PET avidity, and another swollen lymph node was detected near the gastric cardia. No notable abnormalities, including the levels of serum LDH and soluble IL 2 receptor, were found in a blood test.

We obtained written informed consent, and patient anonymity has been preserved.

Pathological findings. Cervical cytology was performed by liquid-based cytology (LBC) using the Sure Path method. The LBC slide demonstrated a necrotic background and scattered atypical cells with high nuclear/cytoplasmic ratios (Fig. 2A). Approximately $80 \%$ of the cells observed in the slide were atypical cells. These cells had almost bare and coarse, hyperchromatic, focally cleaved nuclei with prominent nucleoli using a high-power field (Fig. 2B). Since it lacked adepithelial construction, it was suspected to be a non-epithelial malignant tumor, including malignant lymphoma, by cervical cytology. It was classified as 'other malignancy' according to the Bethesda System 2001. A cervical biopsy was concurrently conducted, and the histological examination revealed diffuse large atypical lymphocyte infiltration with prominent nucleoli and mitosis (Fig. 3). Immunohistochemical analysis showed strong positivity for CD45 and CD20 while CD3 was negative. Further 
A

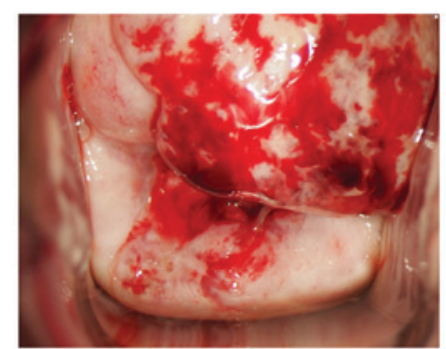

B

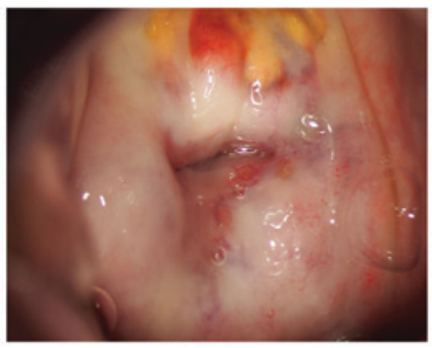

Figure 1. Gross appearance of the uterine cervical tumor. (A) A macroscopic hemorrhagic tumor occupied the anterior lip of the uterine cervix. (B) Following chemotherapy, the cervical tumor completely disappeared.
A

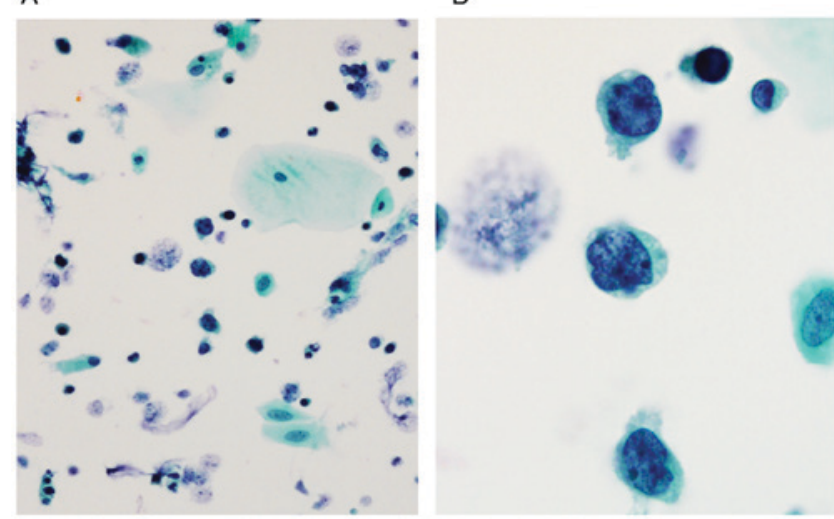

Figure 2. Cytological findings of the uterine cervical tumor by Papanicolaou staining. (A) The liquid-based cytology slide demonstrated a necrotic background and scattered atypical cells with high nuclear/cytoplasmic ratios. Magnification, x200. (B) Atypical cells had almost bare and coarse, hyperchromatic, focally cleaved nuclei with prominent nucleoli that lacked adepithelial construction. Magnification, x1,000.

results from the immunohistochemical analysis were as follows: AE1/3 (-), p40 (-), CEA (-), S-100 (-), CD10 (-), BCL-2 (-). Immunocytochemical staining demonstrated similar findings to the immunohistochemical analysis (Fig. 4). A bone marrow aspiration biopsy revealed normocellular marrow, and no lymphoma cell invasion was observed. Immunophenotyping of the bone marrow aspirate by flow cytometry showed no aberrant antigen expression or monotypic B cells.

Based on these results, the final diagnosis was diffuse large B cell lymphoma (DLBCL) of the uterine cervix, stage IIEA (Ann Arbor classification). The patient received 6 courses of R-CHOP chemotherapy (rituximab, $375 \mathrm{mg} / \mathrm{m}^{2}$ at day 1 ; cyclophosphamide, $750 \mathrm{mg} / \mathrm{m}^{2}$ at day 1 ; doxorubicin, $50 \mathrm{mg} / \mathrm{m}^{2}$ at day 1 ; vincristine, $1.4 \mathrm{mg} / \mathrm{m}^{2}$ at day 1 ; prednisolone $100 \mathrm{mg} /$ body at day 1-5) and achieved complete remission. The cervical tumor disappeared grossly (Fig. 1B), and atypical cells were not present in the Pap smear and biopsy specimens. Complete remission was also confirmed by PET/CT scan and no residual or relapse tumor was found (data not shown).

\section{Discussion}

We encountered a case of malignant lymphoma of the uterine cervix that was presumptively diagnosed by a Pap smear screening.

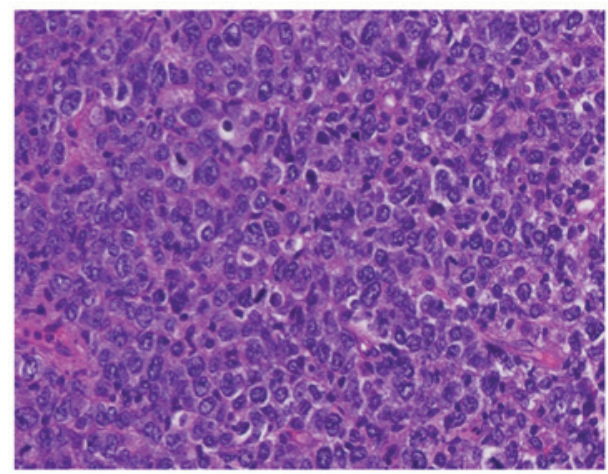

Figure 3. Histological findings of the uterine cervical tumor by haematoxylin and eosin staining. Histological examinations revealed diffuse large atypical lymphocyte infiltration with prominent nucleoli and mitosis. Magnification, $\mathrm{x} 200$.
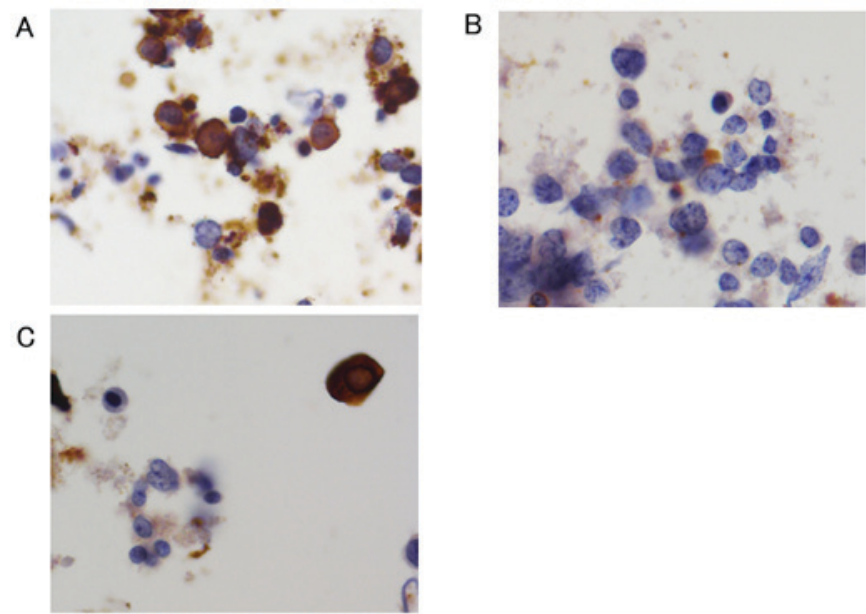

Figure 4. Immunocytochemical analysis. Tumor cells were positive for (A) CD20 but negative for (B) CD3 and (C) AE1/3 (epithelial marker). Magnification, $\mathrm{x} 400$.

Due to its rarity, it is usually difficult to diagnose malignant lymphoma of the uterine cervix by Pap smears. In cervical cytology, the positivity for malignant lymphoma has been reported to be $30-40 \%$ (5-7). This may be due to the fact that while lymphoma cells often infiltrate the cervical stroma, epithelial lining tissues remain preserved (5). In Pap screenings, the classical cytological features of malignant lymphoma are as follows: The presence of a dispersed monomorphic cell population, high nuclear/cytoplasmic ratios, coarse granular nuclear chromatin, focally cleaved nuclei, and the presence of prominent nucleoli (8). The differential diagnosis of malignant lymphoma of the uterine cervix includes chronic cervicitis, lymphoma-like lesions (LLLs), carcinoma, carcinosarcoma, endometrial stromal sarcoma, melanoma (small cell variant), and primitive neuroectodermal tumor (PNET). However, meticulous morphological observations and immunohistochemical analysis can efficiently distinguish malignant lymphoma from these other lesions (9). In our case, the tumor cells were easily obtained due to its extroverted growth, and typical cytological features of malignant lymphoma were observed in cervical cytology. Although approximately $80 \%$ of the cells were atypical cells in the Pap staining of our case, 
precise percentage for the lymphoma cells in the slide has not yet been elucidated to date. Further cytological study seems to be required. In general, diagnosis of B cell lymphoma is based on morphology (H\&E staining) and CD20 positive and CD3 negative, additional immunohistochemical analyses including CD10, BCL-2, and c-Myc may be useful to distinguish histological subtypes.

The clinical diagnosis of cervical lymphoma may be difficult due to the lack of specific symptoms. The most common clinical symptoms have been reported as vaginal bleeding or discharge, and abdominal or pelvic pain. However, some patients were reported to be asymptomatic (9). The correct diagnosis is often delayed and, as a result, the disease is diagnosed at an advanced stage. Our patient was also asymptomatic; however, we were able to detect malignant lymphoma at an early stage through cervical cytology. Therefore, Pap test screenings may be useful for the early diagnosis of malignant lymphoma of the uterine cervix. We did not measure the serum level of such parameters as EBV-DNA and CMV-DNA in the present case. Other hematological malignancies including virus associated ones should be considered when diagnosing malignant lymphoma.

Standard therapy for DLBCL is R-CHOP chemotherapy with or without radiotherapy. In a previous review article, they reported that $30-50 \%$ of patients underwent surgery, which included invasive radical hysterectomy with pelvic lymphadenectomy. Since R-CHOP chemotherapy is the most effective treatment, invasive surgery should be avoided (10).

Despite its rarity, the possibility of malignant lymphoma should be considered when screening for cervical cancers by Pap tests. In addition to conventional morphological observations, it is important to perform immunohistochemical analyses. A rapid and accurate diagnosis can lead to immediate treatment without requiring surgery. The accumulation of more clinical cases is important for obtaining a clearer understanding of the clinicopathological features of this rare tumor.

\section{Acknowledgements}

We thank Daisuke Kawashima and Kanako Inoue for their technical assistance.

\section{Funding}

No funding was received.

\section{Availability of data and materials}

The datasets used and/or analyzed during the current study are available from the corresponding author on reasonable request.

\section{Authors' contributions}

TK, AT and HT diagnosed, investigated and managed the patient. TK, HK and HT determined the medical significance of this case and wrote the manuscript. MA, SI, MI, HY, SN, YT, TF, MG and FE provided advice in managing the patient's treatment and preparing the manuscript.

\section{Ethics approval and consent to participate}

Not applicable.

\section{Consent for publication}

The patient provided written informed consent for the publication of their data.

\section{Competing interests}

The authors confirm that they have no competing interests.

\section{References}

1. Calli AO, Rezanko T, Yigit S and Payzin B: Lymphoma of the cervix: A diagnostic pitfall on cervicovaginal smear. J Cytol 29: 213-215, 2012.

2. Bellevicine C, Zabatta A, Malapelle U, Vetrani A and Troncone G: Diffuse large B-cell extranodal lymphoma of the uterine cervix: An incidental pap smear finding with histological and immunohistochemical correlation. Diagn Cytopathol 42: 644-646, 2014

3. Hanley KZ, Tadros TS, Briones AJ, Birdsong GG and Mosunjac MB: Hematologic malignancies of the female genital tract diagnosed on liquid-based Pap test: Cytomorphologic features and review of differential diagnoses. Diagn Cytopathol 37: 61-67, 2009.

4. Chan JK, Loizzi V, Magistris A, Hunter MI, Rutgers J, DiSaia PJ and Berman ML: Clinicopathologic features of six cases of primary cervical lymphoma. Am J Obstet Gynecol 193: 866-872, 2005.

5. Whitaker D: The role of cytology in the detection of malignant lymphoma of the uterine cervix. Acta Cytol 20: 510-513, 1976.

6. Komaki R, Cox JD, Hansen RM, Gunn WG and Greenberg M: Malignant lymphoma of the uterine cervix. Cancer 54: 1699-1704, 1984.

7. Cardillo MR and Forte F: The diagnostic value of cytology in a case of lymphoma of the uterine cervix. Eur J Gynaecol Oncol 8: 597-602, 1987.

8. Cahill LA, Stastny JF and Frable WJ: Primary lymphoma of the endometrium. A report of two cases diagnosed on cervicovaginal smears. Acta Cytol 41: 533-538, 1997.

9. Lagoo AS and Robboy SJ: Lymphoma of the female genital tract: Current status. Int J Gynecol Pathol 25: 1-21, 2006.

10. Mandato VD, Palermo R, Falbo A, Capodanno I, Capodanno F, Gelli MC, Aguzzoli L, Abrate M and La Sala GB: Primary diffuse large B-cell lymphoma of the uterus: Case report and review. Anticancer Res 34: 4377-4390, 2014. 